\title{
Correlations of Four Genetic Single Nucleotide Polymorphisms in Brain-Derived Neurotrophic Factor with Posttraumatic Stress Disorder
}

\author{
Jun-Cheng Guo ${ }^{1,2 \star}$, Yi-Jun Yang ${ }^{3 \star}$, Min Guo ${ }^{凶}$, Xiao-Dan Wang ${ }^{5}$, Yang Juan ${ }^{5}$, \\ Yun-Suo $\mathrm{Gao}^{6}$, Lin-Qiu Fu' ${ }^{7}$, Xiang-Ling Jiang ${ }^{8}$, Lin-Mei Fu' ${ }^{9}$, and Tao Huang ${ }^{8}$ \\ ${ }^{1}$ Department of Science and Education, Hainan General Hospital, Hainan, China \\ 2Department of Science and Education, Affiliated Hainan Hospital of Nanhua University, Hainan, China \\ ${ }^{3}$ Hainan Province, Haikou People's Hospital, Hospital Dean's Office, Hainan, China \\ ${ }^{4}$ The Psychological Research Center of Hainan General Hospital, Hainan, China \\ ${ }^{5}$ School of Public Health, Hainan Medical College, Hainan, China \\ ${ }^{6}$ Department of Equipment, Hainan General Hospital, Hainan, China \\ ${ }^{7}$ Department of Psychology, Hainan General Hospital, Hainan, China \\ ${ }^{8}$ Department of Clinical Laboratory, Hainan General Hospital, Hainan, China \\ ${ }^{9}$ Medical Center, Hainan General Hospital, Hainan, China
}

Objective To investigate the correlations of four genetic single nucleotide polymorphisms (SNPs) of brain-derived neurotrophic factor (BDNF) with posttraumatic stress disorder (PTSD).

Methods A total of 300 patients with sporadic PTSD and 150 healthy subjects (the control group) were selected according to the diagnostic criteria of PTSD (DSM-IV), and the genotypes of the BDNF SNPs G-712A, C270T, rs6265, and rs7103411 were detected by polymerase chain reaction and direct DNA sequencing to determine intergroup differences in the genotypes and allele frequencies; the $\mathrm{p}$ values were corrected with the permutation test.

Results The genotypes and allele frequencies of the SNPs G-712A, rs6265, and rs7103411 of BDNF showed no significant intergroup differences $(p>0.05)$. However, the genotype and allele frequencies of C270T showed significant differences between the PTSD group and the control group $(\mathrm{p}<0.05)$.

Conclusion The SNP C270T of BDNF may be associated with PTSD. Individuals carrying the polymorphic T allele of C270T may be more likely to suffer from PTSD.

Psychiatry Investig 2018;15(4):407-412

Key Words Brain-derived neurotrophic factor, Posttraumatic stress disorder, Gene polymorphism.

\section{INTRODUCTION}

Posttraumatic stress disorder (PTSD) is a type of reactive mental disorder that develops in individuals after experiencing an unexpected sudden, threatening, and catastrophic se-

Received: November 23, 2016 Revised: June 10, 2017

Accepted: June 17, 2017

$\bowtie$ Correspondence: Min Guo

The Psychological Research Center of Hainan General Hospital, No. 19 Xiuhua Road Xiuying District Haikou City, Hainan Province, 570311, China

Tel: +86 13707587018, Fax: +86898 68663485, E-mail: minguodoc@126.com

*These authors contributed equally to this work.

(a) This is an Open Access article distributed under the terms of the Creative Commons Attribution Non-Commercial License (http://creativecommons.org/licenses/bync/4.0) which permits unrestricted non-commercial use, distribution, and reproduction in any medium, provided the original work is properly cited. vere stress event. ${ }^{1}$ Infamous examples of PTSD include veterans returning from the Vietnam War and the Gulf War, who showed various degrees of stress symptoms such as mental breakdown, which lasted from a few months to several years, and even developed into delayed stress symptoms in some cases. After the 911 event (USA) and the Wenchuan earthquake (China), Western countries and China increased research efforts in gaining a better understanding of PTSD. ${ }^{2}$ At the beginning of this century, the definition and diagnosis of PTSD in the Chinese Classification of Mental Disorders (CCMD-3) were revised to formally conform with international standards. PTSD mainly manifests as three core symptoms: a pathological tendency to reproduce the traumatic experience, emotional numbness and avoidance behavior, and 
excessive vigilance causing irritability. ${ }^{3}$ Long-term PTSD can lead to depression, anxiety, suicidal tendency, schizophrenia, and many other problems, and is most commonly associated with unemployment, family instability, natural disasters, and health emergencies; therefore, PTSD inflicts serious social harm. Among a number of PTSD genetic susceptibility candidate genes identified to date, alterations in the brain-derived neurotrophic factor $(B D N F)$ gene have attracted the most attention. ${ }^{4}$ Animal experiments, and neurobiological and neuroendocrine studies have suggested that BDNF may be related to the occurrence of PTSD, and there is also evidence to suggest an association in humans. First, studies have revealed that BDNF is related to the pathological mechanisms associated with a variety of mental illnesses, and PTSD shows a certain degree of commonality with these psychiatric disorders with respect to the pathogenesis, clinical manifestations, and genetic susceptibility factors. ${ }^{5}$ Second, BDNF is expected to be a new marker for the prognosis and monitoring of the genetic therapy outcomes in a variety of mental disorders and brain traumas. ${ }^{6}$ To investigate the genetic heterogeneity of PTSD, we detected known single nucleotide polymorphism (SNP) loci in BDNF, and compared their differences in 300 patients diagnosed with PTSD and 150 healthy individuals (the control group), who were included in the Hainan PTSD epidemiological survey from August 2012 to March 2016. The relationships of the genotype and allele frequencies of polymorphic sites with PTSD were determined using a case-control design. At present, $B D N F$ is considered to be a candidate causal gene of depression and schizophrenia; however, some reports have shown inconsistent results, which may be affected by certain confounding factors such as age, illness, ethnicity, disease duration, or sample size. The lack of a detailed hierarchical analysis may contribute to this lack of clarity in the conclusions of previous studies, and the results of different studies sometimes lack comparability, even showing opposite effects in some cases. Therefore, the impact of several factors should be carefully considered and investigated when studying the correlations between PTSD and BDNF. To resolve these conflicts, we studied the correlations between $B D N F$ SNPs and PTSD, hoping to further explore the roles of BDNF in the occurrence, development, and prognosis of PTSD, thus providing the basis for better explaining the inconsistency among previous studies.

\section{METHODS}

\section{Subjects}

A total of 300 patients with sporadic PTSD were selected from participants of the epidemiological survey in Hainan Province (August 2012 to March 2016). The patients ranged in age from 18 to 65 years (mean of $39.2 \pm 6.3$ years), including 182 males and 118 females. Two psychiatrists (at least one senior psychiatrist) made the final clinical diagnosis using the PTSD Diagnostic Scales (CAPS), based on the Diagnostic and Statistical Manual of Mental Disorders of the American Psychiatric Association (DSM-IV). Treatment responses were assessed at intake and post-treatment in all patients using a semi structured clinician administered interview (CAPS). ${ }^{7}$ The control group was composed of healthy subjects undergoing a physical examination in Hainan Provincial People's Hospital during the same period, including 79 males and 71 females aged 26-64 years (mean 63.75 \pm 9.14 years). There was no significant difference between the PTSD group and the control group in age ( $\mathrm{t}=-0.9532, \mathrm{p}=-0.3398)$, sex ratio $\left(\chi^{2}=0.3721, \mathrm{p}=\right.$ $0.5412)$, and education background ( $12.65 \pm 5.72$ vs. $3.14 \pm$ 4.77; $\mathrm{t}=0.4848, \mathrm{p}=-0.6288$ ). This study was approved by the Ethics Committee of Hainan Provincial People's Hospital (No. 2012-28), and all the subjects signed the informed consent.

\section{Specimen collection and preservation}

Venous blood $(5-10 \mathrm{~mL})$ was sampled from the elbow of all subjects after they completed the epidemiological investigation and evaluation scale. The blood was then anti-coagulated using ethylenediaminetetraacetic acid and stored in the central laboratory of Hainan Provincial Peoples Hospital at $-20^{\circ} \mathrm{C}$.

\section{DNA extraction and polymerase chain reaction (PCR)}

The whole blood genomic DNA extraction kit (OMEGA bio-tek, Guangzou City Guangdong Province, General agent in China) together with the centrifugal column method was used to extract DNA, the concentration and purity of which met the amplification requirements of PCR.

Primer Premier 5.0 software was used to design the genetic primers for amplification of BDNF SNPs. The PCR primers were as follows: rs6265 upstream 5'-TTTCTCCCTACAGTTC CACCAG-3', downstream 5'-CTCCAAAGGCACTTGAC TACTG-3' (343 bp); rs7103411 upstream 5'-AGGACCCA GTCTCAAAAGCAT-3', downstream 5'- ATTTGGGGGTA AAA GGTCCTCG-3' (421 bp); G-712A upstream 5'-TTTG GTCGGCTAGAAAGCGT-3', downstream 5'-AATAG GAATTCAATCAAGATATAA-3' (142 bp); C270T upstream 5'-CAGAGGAGCCAGCCCGGTGCG-3', downstream 5'-CTCCTGCACCAAGCCCCATTC-3' (213 bp).

Amplifications were conducted in a $50 \mu \mathrm{L}$ reaction system, including $1 \mu \mathrm{L}$ of genomic DNA, $1 \mu \mathrm{L}$ of each primer $(10 \mu \mathrm{M})$, $0.25 \mu \mathrm{L}$ of pfuase $(5 \mathrm{U} / \mu \mathrm{L}), 5 \mu \mathrm{L}$ of $10 \times$ Buffer $[200 \mathrm{mM}$ Tris $\mathrm{HCl}\left(\mathrm{pH} \mathrm{8.8)}, 100 \mathrm{mM} \mathrm{KCl}, 20 \mathrm{mM} \mathrm{MgSO}{ }_{4}, 160 \mathrm{mM}\right.$ $\left(\mathrm{NH}_{4}\right) 2 \mathrm{SO}_{4}, 1 \%$ Triton, and $1 \mathrm{mg} / \mathrm{mL}$ bovine serum albu$\min$ ], $1 \mu \mathrm{L}$ of dNTPs $(10 \mathrm{mM})$, and $40.75 \mu \mathrm{L}$ of deionized wa- 
ter. The PCR conditions of rs6265 and rs7103411 were as follows: pre-denaturation at $98^{\circ} \mathrm{C}$ for $3 \mathrm{~min}$, denaturation at $95^{\circ} \mathrm{C}$ for $1 \mathrm{~min}$, annealing at $60^{\circ} \mathrm{C}$ for $45 \mathrm{sec}$, and extension at $72^{\circ} \mathrm{C}$ for $55 \mathrm{sec}$ for a total of 35 cycles, followed by extension at $72^{\circ} \mathrm{C}$ for $8 \mathrm{~min}$. The PCR conditions of G-712A were: pre-denaturation at $95^{\circ} \mathrm{C}$ for $5 \mathrm{~min}$, denaturation at $95^{\circ} \mathrm{C}$ for $30 \mathrm{sec}$, annealing at $54^{\circ} \mathrm{C}$ for $30 \mathrm{sec}$, and extension at $72^{\circ} \mathrm{C}$ for $55 \mathrm{sec}$ for a total of 35 cycles, followed by extension at $72^{\circ} \mathrm{C}$ for $8 \mathrm{~min}$; the PCR conditions of $\mathrm{C} 270 \mathrm{~T}$ were: pre-denaturation at $95^{\circ} \mathrm{C}$ for $5 \mathrm{~min}$, denaturation at $95^{\circ} \mathrm{C}$ for $30 \mathrm{sec}$, annealing at $60^{\circ} \mathrm{C}$ for $30 \mathrm{sec}$, and extension at $72^{\circ} \mathrm{C}$ for $55 \mathrm{sec}$ for a total of $35 \mathrm{cy}$ cles, followed by extension at $72^{\circ} \mathrm{C}$ for $8 \mathrm{~min}$. Primer synthesis and PCR kits were purchased from Shanghai Generay Co, Ltd.

\section{DNA sequencing}

All amplification products of rs6265, rs7103411, G-712A, and C270T were sequenced by Sangon Biotech (Shanghai) Co, Ltd.

\section{Statistical analysis}

SPSS 11.5 software was used for the $\chi^{2}$ test; the count data are expressed as rates, and the intergroup comparison was made using the $\chi^{2}$ test (f test). The odds ratios (ORs) and 95\% confidence intervals (CIs) were calculated for data with significant differences in four-fold tables, with $\mathrm{p}<0.05$ indicating a statistically significant difference.

\section{RESULTS}

The genotypes and allele frequency distributions of the four polymorphic loci of the BDNF gene (rs6265, rs7103411, G712A, and C270T) were all consistent with Hardy-Weinberg equilibrium. The distributions and statistics of the genotype and allele frequencies of these four polymorphic loci in the PTSD group and control group are shown in Table 1. The genotype and allele frequency distributions of C270T differed significantly between the PTSD group and control group $(\mathrm{p}<$ $0.05)$. The $T$ allele frequency was significantly higher in the PTSD group than in the control group $\left(\chi^{2}=7.221, \mathrm{p}=0.007\right.$, $\mathrm{OR}=0.556,95 \% \mathrm{CI}=0.362-0.8853)$. However, no significant differences between the groups were detected for the genotype and allele frequencies of rs6265, rs7103411, and G-712A $(\mathrm{p}>0.05)$.

\section{DISCUSSION}

PTSD has a high incidence among individuals that have experienced one or more severe stress episodes; however, PTSD will not develop in all individuals that suffer from stressful or traumatic events, and only $7-12 \%$ of such patients will suffer from life-long PTSD.

Some progress has been achieved in understanding the pathogenesis of PTSD. The most widely accepted viewpoint is that PTSD results from an interaction between external environmental factors and individual genetic susceptibility, which mainly manifests in two different forms. The first is changes in the sympathetic adrenal medullae system (SAS) and hypothalamic-pituitary-adrenal axis (HPA) as well as their related neurotransmitters. The affected hormones or neurotransmit-

Table 1. Comparison of the genotypes and allele frequencies of the four SNPs between the PTSD group and the control group [N (\%)]

\begin{tabular}{|c|c|c|c|c|c|c|c|c|}
\hline & $\mathrm{N}$ & & Genotype & & & & OR & $95 \% \mathrm{CI}$ \\
\hline rs7103411 & & $\mathrm{CC}$ & CT & TT & $\mathrm{C}$ & $\mathrm{T}$ & 1.105 & $0.837-1.459$ \\
\hline PTSD & 300 & $96(32.0)$ & $103(34.3)$ & $101(33.7)$ & 295 (49.2) & $305(50.8)$ & & \\
\hline Control & 150 & $50(25)$ & $40(26.7)$ & $60(40.0)$ & $140(46.7)$ & $160(53.3)$ & & \\
\hline$\chi^{2}(\mathrm{P})$ & & & $3.036(0.220)$ & & 0.501 & 479) & & \\
\hline rs6265 & & $\mathrm{AA}$ & AG & GG & A & G & 0.858 & $0.650-1.132$ \\
\hline PTSD & 300 & $87(29.0)$ & $119(39.7)$ & $94(31.3)$ & $293(48.8)$ & 307 (51.2) & & \\
\hline Control & 150 & $53(35.3)$ & $52(34.6)$ & $45(30.0)$ & $158(52.7)$ & $142(47.3)$ & & \\
\hline$\chi^{2}(\mathrm{P})$ & & & $2.005(0.367)$ & & 1.176 & 278) & & \\
\hline G-712A & & AA & AG & GG & A & G & 1.098 & $0.832-1.449$ \\
\hline PTSD & 300 & $93(31.0)$ & $122(40.7)$ & $85(28.3)$ & $308(51.3)$ & $292(48.7)$ & & \\
\hline Control & 150 & $48(35.3)$ & $51(34.7)$ & $51(30.0)$ & $147(49.0)$ & $153(51.0)$ & & \\
\hline$\chi^{2}(\mathrm{P})$ & & & $1.606(0.448)$ & & 0.436 & 509) & & \\
\hline $\mathrm{c} 270 \mathrm{~T}$ & & $\mathrm{CC}$ & CT & TT & $\mathrm{C}$ & $\mathrm{T}$ & 0.556 & $0.362-0.853$ \\
\hline PTSD & 300 & $208(69.3)$ & $81(27.0)$ & $11(3.7)$ & $497(82.8)$ & $103(17.2)$ & & \\
\hline Control & 150 & $124(79.3)$ & $21(17.3)$ & $5(3.3)$ & $269(88.0)$ & $31(12.0)$ & & \\
\hline$\chi^{2}(\mathrm{P})$ & & & $9.897(0.007)$ & & $7.221(0.007)$ & & & \\
\hline
\end{tabular}

PTSD: Posttraumatic Stress Disorder 
ters are mainly glucocorticoids (GC), catecholamines, opioid peptides, glutamate, norepinephrine, or 5-hydroxytryptamine. ${ }^{8}$ The GC levels in the blood and urine tend to be lower in PTSD patients than in healthy individuals. In addition, SAS shows high activity in patients with PTSD, which can not only enhance the negative feedback of GC on the HPA axis but can also increase the secretion of catecholamines (from SAS), thus prompting attention dispersion and high vigilance in such patients, stimulating fear, enhancing memory, and promoting the development and formation of a fear response. The second manifestation of the pathogenesis of PTSD is structural and functional changes in the central nervous system. Recent studies have shown that the amygdala receives external stimuli, judges the importance of the signals, and then induces certain emotions as "resistance, avoidance, or numbness" as well as the release of neurotransmitters and stress hormones in patients with PTSD. Neuroimaging studies have shown that decreased activities in the midfrontal and anterior cingulate regions are associated with increased activities of the amygdala, suggesting that the frontal lobe and the anterior cingulate region network cannot appropriately modulate the activity of the amygdala so as to produce high responsiveness to threats. Imaging studies ${ }^{9}$ have also shown that PTSD patients have a reduced hippocampal volume, and hippocampal injuries are related to the strong fear that is characteristic of these patients. ${ }^{10}$ Neuroelectrophysiologic studies have also shown changes of event-related potential (P300) in PTSD patients, with abnormalities clearly associated with typical PTSD symptoms (repeated experience recurrence, active avoidance, numbness, or over-alertness), indicating that certain PTSD symptoms are associated with recognition and attention deficits.

BDNF was the second neurotrophic factor identified after nerve growth factor (NGF), and its precursor has 247 amino acid residues, which can be translated and processed into one mature alkaline protein consisting of 119 amino acid residues. The internal disulfide bond exists in the form of a dimer in vivo. BDNF is the most abundant neurotrophic factor in the body; it is mainly expressed in the cerebral cortex, hippocampus, and striatum, and plays an important role in brain development, affecting the axial growth and connection of neurons, and mediating the proliferation, differentiation, and survival of neurons. ${ }^{11}$ Its overexpression in the central nervous system plays an important promotive role in the generation of anxiety. ${ }^{12}$

In the central nervous system, BDNF is mainly synthesized in neurons, and is then transported along the axoplasm until the axon terminals; after its release, it mainly plays its roles in target cells through its high-affinity receptor tyrosine kinase B. In addition, BDNF can also be secreted by neuron-action target cells, and then reversely provides nutrition to neurons. Immunohistochemistry has confirmed that BDNF-immunoreactive neurons are widely distributed in the central nervous system of the rat brain, especially in the hippocampus, thalamus, and cortex. In the hippocampus, the BDNF mRNA amount is 20-30 times higher than that of NGF. In the peripheral nervous system, more BDNF is detected in the distal part of the impaired nerve stump. Besides the nerve tissues, a small amount of BDNF is also expressed in the ovary, heart, lung, and skeletal muscle. BDNF is also abundant in human plas$\mathrm{ma}$, and is mainly located in the platelets of the whole blood; however, the source of BDNF in the platelets is not yet clear. Because of its high content in the blood, there has been substantial research on the repair and regeneration roles of BDNF in the peripheral nervous system. Based on the fact that some areas with a weak blood-brain barrier, such as the hypothalamus, may allow for BDNF to pass through the barrier, the mutual inductance of BDNF between the peripheral blood and nerve center cannot be ruled out, and it is necessary to explore whether the changes of BDNF in the peripheral blood can be used as a direct indicator of mental illness or the effects of a particular drug.

BDNF plays a broad and important role in the growth, development, differentiation, regeneration, and function maintenance of many types of neurons, thereby serving as an essential factor for regulating the synaptic plasticity and memory of the hippocampus. Clinical findings have revealed that the memory of fear in PTSD patients is difficult to subside or that the fear that has already gradually subsided can easily recur, suggesting that a fear subsidence disorder may be the key to the occurrence and treatment difficulties of PTSD. Determination of the mechanisms underlying fear subsidence requires further investigation. The hippocampus is an important brain region related to learning and memory, and the longterm potentiation of hippocampal neurons is the neural basis of long-term memory. Blocking the expression of BDNF in the hippocampus can cause the consolidation deficit of longterm memory during its formation process, but does not affect the formation of long-term memory, suggesting that the normal expression of BDNF has significant effects on memory consolidation and long-term maintenance. Injecting recombinant human BDNF into the hippocampus can reverse a long-term memory deficit caused by the protein synthesis inhibitor anisomycin, and after blocking BDNF through injection of BDNF antisense oligonucleotides into the hippocampus of rats with established conditional fear, the fear response to conditional cues weakened; together, these findings indicated that $\mathrm{BDNF}$ is a key factor for the long-term maintenance of fear memory. ${ }^{13,14}$ Andreska et al. ${ }^{15}$ reported that individual glutamatergic presynapses carried up to $90 \%$ of the 
synaptic BDNF immunoreactivity, and only a minor fraction of BDNF molecules was found close to the postsynaptic bars. Our previous study demonstrated that hippocampal neurons can enrich and store high amounts of BDNF in small granules within the mature glutamatergic presynapses, at a principal site of synaptic plasticity. Peters et al. ${ }^{16}$ divided rats into a success group and failure group according to the scores of fear subsidence after conditional fear subsidence training, and found a significantly higher BDNF level in the hippocampus of the success group compared to that in the failure group.

Animal (neurobiological and neuroendocrine) and human studies have suggested that BDNF may be related to the occurrence of PTSD; however, no BDNF gene variant directly related to the incidence of PTSD has been identified to date. ${ }^{17}$ Lee and $\mathrm{Kim}^{18}$ studied 106 Korean PTSD patients and 161 healthy controls and did not find any correlations of the Val$66 \mathrm{Met}$ polymorphism of BDNF with PTSD. Saffari et al. ${ }^{19}$ investigated a small sample of PTSD patients and found that three SNPs (G-712A, C270T, and Val66Met) of BDNF were associated with PTSD susceptibility. With respect to other mental disorders, an animal experiment ${ }^{20}$ also revealed that the levels of BDNF protein and mRNA in rat depression models were decreased, but long-term electroconvulsive treatment could upregulate BDNF protein expression in the hippocampus and frontal cortex. Autopsy studies ${ }^{21}$ also showed that BDNF expression in the hippocampus of depressive patients was significantly lower than that in normal controls, and the expression of BDNF in the dentate gyrus of patients who had received antidepressant treatment was significantly higher than that in untreated patients. In addition, the serum BDNF level in patients with major depression was reported to be significantly lower than that of the normal control group, and antidepressant therapies or non-convulsive electroconvulsive therapy could significantly increase the serum BDNF level, together with significant reduction of the depressive symptoms.

BDNF may mediate several emotional disorders, including PTSD. Hwang et al. ${ }^{22}$ investigated the correlations of the Val66Met (rs6265) polymorphism of BDNF with depression in elderly Han patients. The genotype frequencies significantly differed from those in the control group, and the allele frequency of Met was also significantly higher than that of the control group, suggesting that this polymorphism may be involved in the occurrence of depression and can be considered as a risk factor of depression in Chinese elderly patients. Strauss et al. ${ }^{23}$ reported significant correlations between the $\mathrm{T}$ allele of rs7103411 of BDNF and depression in childhood. There are also many studies about the correlations between the polymorphisms of BDNF and schizophrenia. Spalletta et al. ${ }^{24}$ found that the Val66Met polymorphism of BDNF is related to the symptoms of schizophrenia, especially impulsive behavior, and Zintzaras ${ }^{25}$ reported that subjects carrying the polymorphic T allele of $\mathrm{C} 270 \mathrm{~T}$ exhibited a $63 \%$ greater risk of schizophrenia than those carrying the homozygote C/C genotype, thus supporting the correlations between this polymorphism and schizophrenia. Ji et al. ${ }^{26}$ also reported that BDNF $\mathrm{C} 270 \mathrm{~T}$ is a risk factor of Alzheimer's disease in Asians. Therefore, BDNF is related to the pathogenesis of a variety of mental illnesses. At present, it can be preliminarily speculated that $B D N F$ is a candidate causal gene of depression, schizophrenia, and other mental illnesses, but inconsistent results have also been reported. The genotype distribution frequencies of $\mathrm{C} / \mathrm{T}$ and $\mathrm{T} / \mathrm{T}$ as well as the T allele frequency of C270T of BDNF in children with schizophrenia were found to be significantly higher than those of the control group. ${ }^{26-28}$ Certain differences may also be caused by gene frequency distribution differences among different geographic populations. Since there are many clinical subtypes of PTSD, the nature of subjects enrolled in clinical studies may influence the experimental results, and certain confounding factors such as age, disease, or disease course may also generate a certain impact. False associations may also be produced due to random drift, population heterogeneity, genetic heterogeneity, and population stratification in traditional case-control studies. ${ }^{29,30}$ Therefore, non-meticulous stratification analysis can often reduce the persuasiveness of the conclusions of correlation studies. Moreover, since the results of different studies sometimes lack comparability, and are even conflicting, potential influencing factors are worthy of serious consideration in investigating the correlations between PTSD and BDNF. This study was based on local residents from various cities and counties in Hainan. However, the possible impact of natural disasters in Hainan (such as super typhoon "Wilson" on July 1, 2014) cannot be ruled out; therefore, a certain degree of bias caused by population stratification may also exist. Another limitation of the study is the relatively small sample size and low number of SNPs considered. Therefore, the association of the C270T polymorphism of $B D N F$ with PTSD requires more in-depth analysis at the expression level, as well as using a greater sample size with subjects from different regions and races for confirmation of the present results.

\section{Acknowledgements}

This study was supported by the National Natural Science Foundation of China (81260209); Key Science and Technology Projects of Hainan (ZDXM20130073); International Scientific and Technological Cooperation Projects of Hainan (KJHZ2014-09); Innovation and Entrepreneurship Training Projects of University Students in Hainan (20140119); Applied technology research and development and demonstration and promotion of special, Hainan Province (No: ZDXM2015077); Social development science and technology specific projects, Hainan Province (SF201404). 


\section{REFERENCES}

1. Zhang L, Li XX, Hu XZ. Post-traumatic stress disorder risk and brainderived neurotrophic factor Val66Met. World J Psychiatry 2016;6:1-6.

2. Zhang Z, Ran MS, Li YH, Ou GJ, Gong RR. Prevalence of post-traumatic stress disorder among adolescents after the Wenchuan earthquake in China. Psychol Med 2012;42:1687-1693.

3. Schwarzer R, Cone JE, Li J, Bowler RM. A PTSD symptoms trajectory mediates between exposure levels and emotional support in police responders to 9/11: a growth curve analysis. BMC Psychiatry 2016;16:201.

4. Stratta P, SanitàP, Bonanni RL, de Cataldo SD, Angelucci A, et al. Clinical correlates of plasma brain-derived neurotrophic factor in posttraumatic stress disorder spectrum after a natural disaster. Psychiatry Res 2016;244:165-170.

5. Dauncey MJ. Genomic and epigenomic insights into nutrition and brain disorders. Nutrients 2013;5:887-914.

6. Notaras M, Hill R, van den Buuse M. The BDNF gene Val66Met polymorphism as a modifier of psychiatric disorder susceptibility: progress and controversy. Mol Psychiatry 2015;20:916-930.

7. Blake DD, Weathers FW, Nagy LM, Kaloupek DG, Gusman FD, Charney DS, Keane TM. The development of a clinician-administered PTSD scale. J Trauma Stress 1995;8:75-90.

8. Wimalawansa SJ. Causes and risk factors for post-traumatic stress disorder: the importance of right diagnosis and treatment. Asian J Med Sci 2013;5:29-40.

9. Ramage AE, Laird AR, Eickho SB, Acheson A, Peterson AL, Williamson $\mathrm{DE}$, et al. A coordinate-based meta-analytic model of trauma processing in posttraumatic stress disorder. Hum Brain Mapp 2013;34:33923399.

10. Bae KY, Kim DW, Im CH, Lee SH. Source imaging of P300 auditory evoked potentials and clinical correlations in patients with posttraumatic stress disorder. Prog Neuropsychopharmacol Biol Psychiatry 2011;35:1908-1917.

11. Power MJ, Fyvie C. The role of emotion in PTSD: two preliminary studies. Behav Cogn Psychother 2013;41:162-172.

12. Koven NS, Carr LH. The role of the brain-derived neurotrophic factor C270T polymorphism in executive functioning. J Applied Med Sci 2013; 2:25-41.

13. Lee B, Sur B, Yeom M, Shim I, Lee H, Hahm DH. Effects of systemic administration of ibuprofen on stress response in a rat model of post-traumatic stress disorder. Korean J Physiol Pharmacol 2016;20:357-366.

14. Li RH, Fan M, Hu MS, Ran MS, Fang DZ. Reduced severity of posttraumatic stress disorder associated with Val allele of Val66Met polymorphism at brain-derived neurotrophic factor gene among China adolescents after Wenchuan earthquake. Psychophysiology 2016;53: 705-711.

15. Andreska T, Aufmkolk S, Sauer M, Blum R. High abundance of BDNF within glutamatergic presynapses of cultured hippocampal neurons. Front Cell Neurosci 2014;8:107.

16. Peters J, Dieppa-Perea LM, Melendez LM, Quirk GJ. Induction of Fear
Extinction with Hippocampal-Infralimbic BDNF. Science 2010;328: 1288-1290.

17. Zhang L, Benedek DM, Fullerton CS, Forsten RD, Naifeh JA, Li XX, et al. PTSD risk is associated with BDNF Val66Met and BDNF overexpression. Mol Psychiatry 2014;19:8-10.

18. Lee HY, Kim YK. Plasma brain-derived neurotrophic factor as peripheral marker for the action mechanism of antidepressant. Neuropsychobiology 2008;57:194-199.

19. Saffari S, Abrari K, Rezaei A, Rashidy-Pour A, Goudarzi I, Salmani ME. Correlation of fear memory in a PTSD animal model and hippocampal BDNF in response to $\beta$-estradiol treatment. Am Pol Sci Assoc 2015;85:61-65.

20. Boiocchi C, Maggioli E, Zorzetto M, Sinforiani E, Cereda C, Ricevuti G, Cuccia M. Brain-derived neurotrophic factor gene (BDNF) variants and Alzheimer's disease: an association study in an AD Italian population. Rejuvenation Res 2012;16:57-66.

21. Sullivan GM, Neria Y. Pharmacotherapy of PTSD: current status and controversies. Psychiatr Ann 2009;39:342-347.

22. Hwang JP, Tsai SJ, Hong CJ, Yang CH, Lirng JF, Yang YM. The Val66Met polymorphism of the brain-derived neurotrophic factor gene is associated with geriatric depression. Neurobiol Aging 2006;27:1834-1837.

23. Strauss J, Barr CL, George CJ, Devlin B, Vetró A, Kiss E, et al. Brain-derived neurotrophic factor variants are associated with childhood-onset mood disorder: confirmation in a Hungarian sample. Mol Psychiatry 2005;10:861-867.

24. Spalletta G, Morris DW, Angelucci F, Rubino IA, Spoletini I, Bria P, et al. BDNF Val66Met polymorphism is associated with aggressive behavior in schizophrenia. Eur Psychiatry 2010;25:311-313.

25. Zintzaras E. Brain-derived neurotrophic factor gene polymorphisms and schizophrenia: a meta analysis. Psychiatr Genet 2007;17:69-75.

26. Ji H, Dai D, Wang Y, Jiang D, Zhou X, Lin P, et al. Association of BDNF and BCHE with Alzheimer's disease: Meta-analysis based on 56 genetic case-control studies of 12,563 cases and 12,622 controls. Exp Ther Med 2015;9:1831-1840.

27. Mirowska-Guzel D, Gromadzka G, Mendel T, Janus-Laszuk B, Dzierka J, Sarzynska-Dlugosz I, et al. Impact of BDNF -196 G>A and BDNF $-270 \mathrm{C}>\mathrm{T}$ polymorphisms on stroke rehabilitation outcome: sex and age differences. Top Stroke Rehabil 2014;21(Suppl 1):S33-S41.

28. Bruenig D, Lurie J, Morris CP, Harvey W, Lawford B, Young RM, et al. A case-control study and meta-analysis RevealBDNFVal66Met is a possible risk factor for PTSD. Neural Plast 2016;2016:6979435.

29. Zhang KY, Song MF, Tao YH, Zhu CY, Zhang L, Dong JZ, et al. Association between brain-derived neurotrophic factor gene C270T polymorphisms and the clinical efficacy of resperidone in schizophrenia. Chin J Clin Pharmacol 2016;1:21-23.

30. Guo JC, Tian ZL, Wang XD, Guo M, Guo M, Li MY, et al. Analysis of psychological stress factors in residents affected by typhoon Rammasun structural equation model. Asian Pac J Trop Med 2016;9:876-880. 